PROCEEDINGS OF THE

AMERICAN MATHEMATICAL SOCIETY

Volume 134, Number 4, Pages 1153-1160

S 0002-9939(05)08050-0

Article electronically published on September 28, 2005

\title{
COMPLETELY MONOTONIC FUNCTIONS INVOLVING THE GAMMA AND $q$-GAMMA FUNCTIONS
}

\author{
ARCADII Z. GRINSHPAN AND MOURAD E. H. ISMAIL \\ (Communicated by Carmen C. Chicone)
}

\begin{abstract}
We give an infinite family of functions involving the gamma function whose logarithmic derivatives are completely monotonic. Each such function gives rise to an infinitely divisible probability distribution. Other similar results are also obtained for specific combinations of the gamma and $q$-gamma functions.
\end{abstract}

\section{INTRODUCTION}

We first recall some definitions and basic facts. A function $f$ is completely monotonic if for all $n,(-1)^{n} f^{(n)}(x) \geq 0$ on $(0, \infty)$; see Feller [12] and Widder [27] for properties of completely monotonic functions. Bernstein's theorem asserts that $f$ is completely monotonic if and only if $f(x)=\int_{\mathbb{R}} e^{-x t} d \mu(t)$ where $\mu$ is a positive measure supported on a subset of $[0, \infty)$. A probability measure is called infinitely divisible if for every $n, n=1,2, \ldots$, there exists a probability measure $\mu_{n}$ such that $\mu$ is an $n$-fold convolution of $\mu_{n}$. The Lévy-Khinchin theorem characterizes infinitely divisible measures supported on a subset of $[0, \infty)$ as those measures whose Laplace transform is of the form $f(x)=e^{-h(x)}$ and $h^{\prime}$ is completely monotonic. This holds in a wider sense without the standard normalization $f(0)=1$, and also for $f(0)=\infty$ (cf. [8]). We remind the reader that any function $f(x)=e^{-h(x)}$ is completely monotonic if $h^{\prime}$ is completely monotonic. We call such functions $f(x)$ logarithmically completely monotonic [8, 24, 25]. Berg [8] points out that these functions are the same as those studied by Horn 20, under the name infinitely divisible completely monotonic functions. The subject of deriving inequalities for combinations of gamma functions is an old subject and some of the early works are by Kazarinoff [23], Watson [26], and Gautschi [14, 15]. These papers provide bounds for quotients of products of gamma functions. In the 1980s Ismail et al., [21], 9], realized that some of the inequalities are consequences of the complete monotonicity of functions of similar structure. We continue to witness progress in this area as can be seen from the more recent works [1, 2, 3, 4, 6, 22] (see also [5]). This paper is a further contribution to the subject.

Received by the editors April 12, 2004 and, in revised form, October 28, 2004 and November 9, 2004.

2000 Mathematics Subject Classification. Primary 33B15; Secondary 26A48.

Key words and phrases. Gamma function, $q$-gamma function, completely monotonic functions, inequalities.

The second author's research was partially supported by NSF grant DMS 99-70865. 
In Section 2 we prove Theorems 1.1 and 1.2 below. Before stating them we need some notation. Let $S_{n}$ be the symmetric group over $n$ symbols, $a_{1}, a_{2}, \ldots, a_{n}$. Let $O_{n}$ and $E_{n}$ be the sets of odd and even permutations over $n$ symbols, respectively. Finally, let $P_{n, k}(k=1, \ldots, n)$ be the set of all vectors $\mathbf{m}=\left(m_{1}, \ldots, m_{k}\right)$ whose components are natural numbers such that $1 \leq m_{\nu}<m_{\mu} \leq n$ for $1 \leq \nu<\mu \leq k$; and let $P_{n, 0}$ be the empty set.

Theorem 1.1. Let $a_{1}>a_{2}>\cdots>a_{n}>0$ and define

$$
F(x)=\frac{\prod_{\sigma \in E_{n}}\left[\Gamma\left(x+a_{\sigma(2)}+2 a_{\sigma(3)}+\cdots+(n-1) a_{\sigma(n)}\right)\right]}{\prod_{\sigma \in O_{n}}\left[\Gamma\left(x+a_{\sigma(2)}+2 a_{\sigma(3)}+\cdots+(n-1) a_{\sigma(n)}\right)\right]} .
$$

Then $F\left(x-a_{2}-2 a_{3}-\cdots-(n-1) a_{n}\right)$ is logarithmically completely monotonic.

Observe that

$$
\sum_{k=1}^{n}(k-1)\left(a_{\sigma(k)}-a_{k}\right) \geq 0
$$

holds for any permutation $\sigma$ when $a_{1}>a_{2}>\cdots>a_{n}>0$. This follows from Theorem 368 of Section 10.2 in Hardy, Littlewood, and Pólya [19]. Therefore $F\left(x-a_{2}-2 a_{3}-\cdots-(n-1) a_{n}\right)$ is defined for $x>0$.

Theorem 1.2 below is more general than Theorem 1.1. It is also a generalization of a result in Bustoz and Ismail [9] which corresponds to the case $n=2$ in Theorem 1.2. The Ismail-Bustoz result is that the function

$$
g(x)=\frac{\Gamma(x) \Gamma(x+a+b)}{\Gamma(x+a) \Gamma(x+b)}
$$

is completely monotonic for any $a, b \geq 0$.

Theorem 1.2. For any $a_{k}>0(k=1, \ldots, n)$, define

$$
F_{n}(x)=\frac{\Gamma(x) \prod_{k=1}^{[n / 2]}\left[\prod_{\mathbf{m} \in P_{n, 2 k}} \Gamma\left(x+\sum_{j=1}^{2 k} a_{m_{j}}\right)\right]}{\prod_{k=1}^{[(n+1) / 2]}\left[\prod_{\mathbf{m} \in P_{n, 2 k-1}} \Gamma\left(x+\sum_{j=1}^{2 k-1} a_{m_{j}}\right)\right]} .
$$

Then $F_{n}(x)$ is logarithmically completely monotonic. Any product of functions of the type (1.3) with different parameters $a_{k}$ is logarithmically completely monotonic as well.

In addition, note that $F_{n}(x)=F_{n-1}(x) / F_{n-1}\left(x+a_{n}\right)$ if $F_{n}$ and $F_{n-1}$ are defined in (1.3) by the same parameters $a_{k}$. Also $\lim F_{n}(x)_{a_{n} \rightarrow \infty}=F_{n-1}(x), n>2$.

Here are some consequences of Theorem 1.2.

Corollary 1.3. For $a>0$ and $n=1,2, \ldots$, let

$$
L_{n}(x)=\frac{\prod_{k=0}^{[n / 2]} \Gamma^{\left(\begin{array}{c}
n \\
2 k
\end{array}\right)}(x+2 k a)}{\prod_{k=1}^{[(n+1) / 2]} \Gamma^{\left(\begin{array}{c}
{ }^{n}-1 \\
2 k-1
\end{array}\right)}(x+(2 k-1) a)} .
$$

Then $L_{n}(x)$ is logarithmically completely monotonic.

Corollary 1.4. The function

$$
\frac{\Gamma(x) \Gamma(x+\alpha+\beta) \Gamma(x+\lambda+\alpha) \Gamma(x+\lambda+\beta)}{\Gamma(x+\alpha) \Gamma(x+\beta) \Gamma(x+\lambda) \Gamma(x+\lambda+\alpha+\beta)}
$$

with nonnegative parameters $\alpha, \beta, \lambda$ is logarithmically completely monotonic. 
Corollary 1.5. Let

$$
\begin{gathered}
f(x)=\frac{\Gamma(x) \Gamma(x+\alpha+\beta+\gamma+\delta)}{\Gamma(x+\lambda+\alpha+\beta+\gamma+\delta) \Gamma(x+\lambda)} \\
\times \frac{\Gamma(x+\lambda+\alpha+\gamma) \Gamma(x+\lambda+\beta+\delta)}{\Gamma(x+\alpha+\delta) \Gamma(x+\beta+\gamma)} .
\end{gathered}
$$

Then $f(x)$ is logarithmically completely monotonic when $\min \{\lambda, \alpha, \beta, \gamma, \delta\} \geq 0$ and $(\beta-\alpha)(\delta-\gamma)>0$.

Note that the function $F_{n}(x)$ reduces to $L_{n}(x)$ when $a_{k}=a(k=1, \ldots, n)$. Function (1.5) is a function of the type (1.3) for $n=3$. According to our observation, the limiting case $\lambda \rightarrow \infty$ of the function appearing in (1.5) is function (1.3) for $n=2$, i.e. function (1.2) of Bustoz and Ismail [9]. Function (1.6) is a product of two functions of the type (1.3), namely: $F_{2}(x+\alpha+\gamma)$ with $a_{1}=\delta-\gamma$ and $a_{2}=\beta-\alpha$ (if $\delta>\gamma$ and $\beta>\alpha$ ), and $F_{3}(x)$ with $a_{1}=\alpha+\gamma, a_{2}=\beta+\delta$, and $a_{3}=\lambda$. There is an interesting connection between function (1.6) and the bi-Hermitian form of the general inequalities for fractional integrals and some values of the gamma function [17. (see also [16, 18, for related results).

The $q$-gamma function is, 13,

$$
\Gamma_{q}(z):=(1-q)^{1-z} \prod_{n=0}^{\infty} \frac{1-q^{n+1}}{1-q^{n+z}}, \quad 0<q<1 .
$$

The $q$-gamma function extends the gamma function in the sense that

$$
\lim _{q \rightarrow 1^{-}} \Gamma_{q}(z)=\Gamma(z) .
$$

There are few known monotonicity properties of the $q$-gamma function; see 21] and 222. One of the results proved in Ismail and Muldoon's work 22] is that both

$$
\frac{\prod_{k=1}^{n} \Gamma_{q}\left(x+a_{k}\right)}{\Gamma_{q}^{n}(x+\bar{a})}, \quad \bar{a}:=\frac{1}{n} \sum_{k=1}^{n} a_{k},
$$

and

$$
\frac{\Gamma_{q}^{n-1}(x) \Gamma_{q}\left(x+a_{1}+a_{2}+\cdots+a_{n}\right)}{\prod_{k=1}^{n} \Gamma_{q}\left(x+a_{k}\right)}
$$

are logarithmically completely monotonic for $0<q \leq 1$ and $a_{k}>0$. In Section 3 we extend Theorems 1.1 and 1.2 to the $q$-gamma function and generalize the results from $[22$.

\section{Proofs of Theorems 1.1 AND 1.2}

A basic tool used in the proofs of Theorems 1.1 and 1.2 is the integral representation

$$
\frac{\Gamma^{\prime}(z)}{\Gamma(z)}=-\gamma+\int_{0}^{1} \frac{1-t^{z-1}}{1-t} d t, \quad \Re z>0,
$$

where $\gamma$ is the Euler constant, [11, p. 16]. 
Lemma 2.1. Let $\alpha_{k}$ and $\beta_{k}(k=1, \ldots, n)$ be real numbers such that $\sum_{k=1}^{n} \alpha_{k}=0$ and $\beta_{k} \geq 0$ for all $k$. Then

$$
u(x)=\prod_{k=1}^{n} \Gamma^{\alpha_{k}}\left(x+\beta_{k}\right)
$$

is logarithmically completely monotonic if and only if

$$
v(t)=\sum_{k=1}^{n} \alpha_{k} t^{\beta_{k}} \geq 0
$$

for all $t \in(0,1]$.

Note that functions of the form (2.3) are called Müntz polynomials. Also note that Lemma 2.1 can be generalized for functions of the type

$$
u(x)=\exp \left\{\int_{E} \log \Gamma[x+\beta(y)] d \alpha(y)\right\},
$$

where $\alpha(y)$ is a finite measure on a set $E, \alpha(E)=0$, and a nonnegative-valued function $\beta(y)$ satisfies the following condition: $\int_{E} t^{\beta(y)} d \alpha(y) \geq 0$ for all $t \in(0,1]$. In the next section a similar remark can be made on a generalization of Lemma 3.1 .

Proof of Lemma 2.1. Since $u(x)>0$ on $(0, \infty)$ we let $h(x)=-\log u(x)$. Clearly the integral representation (2.1) implies

$$
h^{\prime}(x)=\int_{0}^{1} \frac{t^{x-1}}{1-t} v(t) d t .
$$

Proof of Theorem 1.1. We use Lemma 2.1. The function $v(t)$ defined by (2.3) equals

$$
\sum_{\sigma \in E_{n}} t^{a_{\sigma(2)}+2 a_{\sigma(3)}+\cdots+(n-1) a_{\sigma(n)}}-\sum_{\sigma \in O_{n}} t^{a_{\sigma(2)}+2 a_{\sigma(3)}+\cdots+(n-1) a_{\sigma(n)}} .
$$

Hence

$$
v(t)=\sum_{\sigma \in S_{n}} \operatorname{sign}(\sigma) t^{a_{\sigma(2)}} t^{2 a_{\sigma(3)}} \ldots t^{(n-1) a_{\sigma(n)}}
$$

or

$$
v(t)=\mathbf{V}\left(t^{a_{1}}, t^{a_{2}}, \ldots, t^{a_{n}}\right),
$$

where $\mathbf{V}$ stands for a Vandermonde determinant. Therefore

$$
v(t)=\prod_{1 \leq j<k \leq n}\left[t^{a_{k}}-t^{a_{j}}\right] \geq 0
$$

for $t \in(0,1]$.

Proof of Theorem 1.2. In this case

$$
v(t)=\sum_{k=0}^{n}(-1)^{k} \sum_{\mathbf{m} \in P_{n, k}} t^{\sum_{j=1}^{k} a_{m_{j}}} .
$$


Thus

$$
v(t)=1-\sum_{j=1}^{n} t^{a_{j}}+\sum_{\mathbf{m} \in P_{n, 2}} t^{a_{m_{1}}+a_{m_{2}}}-\cdots+(-1)^{n} t^{\sum_{j=1}^{n} a_{j}},
$$

and we have $v(t)=\prod_{k=1}^{n}\left(1-t^{a_{k}}\right) \geq 0$.

\section{3. $q$-GAMMA FUNCTION RESULTS}

In Lemma 3.1 we use functions of the form (2.3) as they have already been used in Lemma 2.1 .

Lemma 3.1. Let $\alpha_{k}$ and $\beta_{k}(k=1, \ldots, n)$ be real numbers such that $\sum_{k=1}^{n} \alpha_{k}=0$ and $\beta_{k} \geq 0$ for all $k$. Then

$$
u(x, q)=\prod_{k=1}^{n} \Gamma_{q}^{\alpha_{k}}\left(x+\beta_{k}\right)
$$

is logarithmically completely monotonic in $x(q \in(0,1))$ if

$$
v(t)=\sum_{k=1}^{n} \alpha_{k} t^{\beta_{k}} \geq 0
$$

for all $t \in(0,1]$.

The analogue of (2.1) to be used here is

$$
\frac{\partial_{x} \Gamma_{q}(x)}{\Gamma_{q}(x)}=-\log (1-q)+\log q \sum_{m=0}^{\infty} \frac{q^{m+x}}{1-q^{m+x}}
$$

which follows from (1.7).

Proof of Lemma 3.1. Clearly

$$
\begin{aligned}
& \frac{\partial_{x} u(x, q)}{u(x, q)}=\sum_{k=1}^{n} \alpha_{k} \frac{\partial_{x} \Gamma_{q}\left(x+\beta_{k}\right)}{\Gamma_{q}\left(x+\beta_{k}\right)} \\
& =\log q \sum_{k=1}^{n} \alpha_{k} \sum_{m=0}^{\infty} \frac{q^{m+x+\beta_{k}}}{1-q^{m+x+\beta_{k}}} \\
& =\log q \sum_{m=0}^{\infty} \sum_{l=1}^{\infty} q^{(m+x) l} v\left(q^{l}\right) \leq 0,
\end{aligned}
$$

which is a discrete analogue of the integral representation arising in the proof of Lemma 2.1. The rest of the proof is obvious.

The functions in (1.9) and (1.10) satisfy the condition of Lemma 3.1. Indeed, in the first case $v(t)=\sum_{k=1}^{n} t^{a_{k}}-n t^{\bar{a}}$, and one can use the arithmetic mean-geometric mean inequality to show that $v(t)$ is not negative on $t \in(0,1]$. In the second case $v(t)=n-1+t^{\sum_{k=1}^{n} a_{k}}-\sum_{k=1}^{n} t^{a_{k}}$. The nonnegativity of this function on $t \in(0,1]$ is a consequence of two facts: $v^{\prime}(t)<0$ and $v(1)=0$.

Theorem 3.2. Let $a_{1}>a_{2}>\cdots>a_{n}>0$ and define

$$
F(x, q)=\frac{\prod_{\sigma \in E_{n}}\left[\Gamma_{q}\left(x+a_{\sigma(2)}+2 a_{\sigma(3)}+\cdots+(n-1) a_{\sigma(n)}\right)\right]}{\prod_{\sigma \in O_{n}}\left[\Gamma_{q}\left(x+a_{\sigma(2)}+2 a_{\sigma(3)}+\cdots+(n-1) a_{\sigma(n)}\right)\right]} .
$$


Then $F\left(x-a_{2}-2 a_{3}-\cdots-(n-1) a_{n}, q\right)$ is a logarithmically completely monotonic function of $x$.

Proof of Theorem 3.2. The proof is similar to the proof of Theorem 1.1, but now we use Lemma 3.1. Clearly the function $v(t)$ in this case equals the Vandermonde determinant $\mathbf{V}\left(t^{a_{1}}, t^{a_{2}}, \ldots, t^{a_{n}}\right)$. Thus

$$
v(t)=\prod_{1 \leq j<k \leq n}\left[t^{a_{k}}-t^{a_{j}}\right] \geq 0 .
$$

Theorem 3.3. Let $a_{k}>0(k=1, \ldots, n)$. Define

$$
F_{n}(x, q)=\frac{\Gamma_{q}(x) \prod_{k=1}^{[n / 2]}\left[\prod_{\mathbf{m} \in P_{n, 2 k}} \Gamma_{q}\left(x+\sum_{j=1}^{2 k} a_{m_{j}}\right)\right]}{\prod_{k=1}^{[(n+1) / 2]}\left[\prod_{\mathbf{m} \in P_{n, 2 k-1}} \Gamma_{q}\left(x+\sum_{j=1}^{2 k-1} a_{m_{j}}\right)\right]} .
$$

Then $F_{n}(x, q)$ is a logarithmically completely monotonic function of $x$. Any product of functions (3.4) with different parameters $a_{k}$ is a logarithmically completely monotonic function of $x$.

Proof of Theorem 3.3. The proof is similar to the proof of Theorem 1.2. We use Lemma 3.1. The function $v(t)=\prod_{k=1}^{n}\left(1-t^{a_{k}}\right) \geq 0$.

Corollary 3.4. For $a>0$ and $n=1,2, \ldots$, let

$$
L_{n}(x, q)=\frac{\left.\prod_{k=0}^{[n / 2]} \Gamma_{q}^{(2 k}\right)(x+2 k a)}{\left.\prod_{k=1}^{[(n+1) / 2]} \Gamma_{q}^{(2 k-1}\right)(x+(2 k-1) a)} .
$$

Then $L_{n}(x, q)$ is a logarithmically completely monotonic function of $x$.

Corollary 3.5. The function

$$
\frac{\Gamma_{q}(x) \Gamma_{q}(x+\alpha+\beta) \Gamma_{q}(x+\lambda+\alpha) \Gamma_{q}(x+\lambda+\beta)}{\Gamma_{q}(x+\alpha) \Gamma_{q}(x+\beta) \Gamma_{q}(x+\lambda) \Gamma_{q}(x+\lambda+\alpha+\beta)}
$$

with nonnegative parameters $\alpha, \beta, \lambda$ is logarithmically completely monotonic in $x$.

Corollary 3.6. Let

$$
\begin{gathered}
f(x, q)=\frac{\Gamma_{q}(x) \Gamma_{q}(x+\alpha+\beta+\gamma+\delta)}{\Gamma_{q}(x+\lambda+\alpha+\beta+\gamma+\delta) \Gamma_{q}(x+\lambda)} \\
\times \frac{\Gamma_{q}(x+\lambda+\alpha+\gamma) \Gamma_{q}(x+\lambda+\beta+\delta)}{\Gamma_{q}(x+\alpha+\delta) \Gamma_{q}(x+\beta+\gamma)} .
\end{gathered}
$$

Then $f(x, q)$ is logarithmically completely monotonic in $x$ when $\min \{\lambda, \alpha, \beta, \gamma, \delta\} \geq$ 0 and $(\beta-\alpha)(\delta-\gamma)>0$.

\section{ACKNOWLEDGEMENT}

The authors are grateful to the referee for many helpful comments and for informing us of several references including [6] and [8]. 


\section{REFERENCES}

[1] H. Alzer, On some inequalities for the gamma and psi functions, Math. Comp. 66 (1997), 373-389. MR1388887 (97e:33004)

[2] H. Alzer, Inequalities for the gamma function, Proc. Amer. Math. Soc. 128 (1999), 141-147. MR 1622757 (2000c:33003)

[3] H. Alzer, On a gamma function inequality of Gautschi, Proc. Edinb. Math. Soc. (2) 45 (2002), 589-600. MR1933741 (2003g:33001)

[4] H. Alzer, On Ramanujan's double inequality for the gamma function, Bull. Lond. Math. Soc. 35 (2003), 601-607. MR1989488 (2004g:33004)

[5] H. Alzer, On Gautschi's harmonic mean inequality for the gamma function, J. Comp. Appl. Math. 157 (2003), 243-249. MR1996881 (2004h:33005)

[6] H. Alzer and C. Berg, Some classes of completely monotonic functions, II, The Ramanujan Journal, to appear.

[7] G. E. Andrews, R. A. Askey, and R. Roy, Special Functions, Cambridge University Press, Cambridge, 1999. MR.1688958 (2000g:33001)

[8] C. Berg, Integral representation of some functions related to the gamma function, Mediterranean J. Math., to appear.

[9] J. Bustoz and M. E. H. Ismail, On gamma function inequalities, Math. Comp. 47 (1986), 659-667. MR0856710 (87m:33002)

[10] J. Dutka, On some gamma function inequalities, SIAM J. Math. Anal. 16 (1985), 180-185. MR0772877 (86e:33002)

[11] A. Erdelyi, W. Magnus, F. Oberhettinger, and G. F. Tricomi, Higher Transcendental Functions, V. 1, McGraw-Hill, New York, 1953. MR0058756 (15:419i)

[12] W. Feller, An Introduction to Probability Theory and Its Applications, V. 2, Academic Press, New York, 1966. MR0210154(35:1048)

[13] G. Gasper and M. Rahman, Basic Hypergeometric Series, Cambridge University Press, Cambridge, 1990. MR1052153 (91d:33034)

[14] W. Gautschi, Some elementary inequalities relating to the gamma and incomplete gamma function, J. Math. Phys. 38 (1959), 77-81. MR0103289 (21:2067)

[15] W. Gautschi, A harmonic mean inequality for the gamma function, SIAM J. Math. Anal. 5 (1974), 278-281. MR0350077 (50:2570)

[16] A. Z. Grinshpan, Inequalities generated by the gamma function, Adv. in Appl. Math. 29 (1) (2002), 115-125. MR1921547 (2003g:30002)

[17] A. Z. Grinshpan, General inequalities, consequences and applications, Adv. in Appl. Math. 34 (1) (2005), 71-100, available online at www.sciencedirect.com (2004). MR2102276 (2005g:26027)

[18] A. Z. Grinshpan, An integral recursive inequality and applications, The Ramanujan Journal, to appear.

[19] G. H. Hardy, J. E. Littlewood, and G. Pólya, Inequalities, 2nd ed., University Press, Cambridge, 1952. MR0046395(13:727e)

[20] R. A. Horn, On infinitely divisible matrices, kernels and functions, Z. Wahrscheinlichkeitstheorie und Verw. Geb. 8 (1967), 219-230. MR0217836 (36:925)

[21] M. E. H. Ismail, L. Lorch, and M. E. Muldoon, Completely monotonic functions associated with the gamma function and its q-analogue, J. Math Anal. Appl. 116 (1986), 1-9. MR0837337 (88b:33002)

[22] M. E. H. Ismail and M. E. Muldoon, Inequalities and monotonicity properties for the gamma and q-gamma functions, in Approximation and Computation, Internat. Ser. Numer. Math. 119, R. Zahar, ed., Birkhäuser, Boston, MA, 1994, 309-323. MR1333625 (96g:33001)

[23] D. K. Kazarinoff, On Wallis' formula, Edinburgh Math. Notes 40 (1956), 19-21. MR0082501 $(18: 560 \mathrm{~g})$

[24] Feng Qi, Bai-Ni Guo, and Chao-Ping Chen, Some completely monotonic functions involving the gamma and polygamma functions, RGMIA Res. Rep. Coll. 7 (1), Art. 5 (2004); available online at http://rgmia.vu.edu.au/v7n1.html.

[25] Feng Qi and Bai-Ni Guo, Complete monotonicities of functions involving the gamma and digamma functions, RGMIA Res. Rep. Coll. 7 (1), Art. 8 (2004); available online at http://rgmia.vu.edu.au/v7n1.html. 
[26] G. N. Watson, A note on gamma functions, Proc. Edinburgh Math. Soc. (2) 11 1958/59 Edinburgh Math. Soc. Notes, No 42 (misprinted 41), 7-9. MR0117358 (22:8138)

[27] D. V. Widder, The Laplace Transform, Princeton University Press, Princeton, 1941. MR0005923 (3:232d)

Department of Mathematics, University of South Florida, Tampa, Florida 33620-5700

E-mail address: azg@math.usf.edu

Department of Mathematics, University of Central Florida, Orlando, Florida 32816

E-mail address: ismail@math.ucf.edu 